\title{
A NOTE ON EXTREME ELEMENTS IN $A_{0}(K, E)$
}

\section{SHARIR ${ }^{1}$}

ABSTRACT. We give a short and simple proof to a theorem of Fakhoury, characterizing extreme elements in the unit ball of $A_{0}(K, E)$.

Let $V$ be a Banach space whose dual is an $L^{1}$-space. Denote by $K$ the unit ball of $V^{*}$ equipped with the $w^{*}$-topology. Let $E$ be a Banach space. $S(E)$ will denote its closed unit ball and ext $S(E)$ the set of extreme points in $S(E) . A_{0}(K, E)$ will be the Banach space of all the symmetric affine functions from $K$ into $E$, continuous in the $w^{*}$-topology on $K$, and in the norm topology on $E$. Fakhoury has shown in [1] that if $E$ has certain properties, then $f \in A_{0}(K, E)$ is extreme in $S\left(A_{0}(K, E)\right)$ if and only if $f($ ext $K) C$ ext $S(E)$. This result bears immediate characterization of extreme compact operators (if $E=F^{*}$, then $A_{0}(K, E)$ is precisely the space of compact operators from $F$ into $V$ ), and generalizes similar results of Lazar [2]. By observing a simple property of spaces having the 3.2.I.P. (cf. [4] for a proper definition), and by using a selection theorem of Lazar and Lindenstrauss [3], we are able to prove Fakhoury's result in a very simple and direct way.

Lemma. Let $E$ be a Banach space having the 3.2.I.P. and let $x, y \in S(E), a \in E$, such that $x \pm a \in S(E)$. Then there exists an element $b \in E$, such that $y \pm b \in S(E)$, and $\|b-a\| \leq\|y-x\|$.

Proof. Define three closed balls in $E$ :

$$
\begin{aligned}
& S_{1}=\{b \in E ;\|b-y\| \leq 1\}, \\
& S_{2}=\{b \in E ;\|b+y\| \leq 1\}, \\
& S_{3}=\{b \in E ;\|b-a\| \leq\|y-x\|\} .
\end{aligned}
$$

These balls intersect in pairs, for $0 \in S_{1} \cap S_{2}, y+a-x \in S_{1} \cap S_{3}$ and

Received by the editors August 15, 1973.

AMS (MOS) subject classifications (1970). Primary 47D20, 46E 15, 46E30, 47B05.

${ }^{1}$ This paper is a part of the author's Ph.D. Thesis, prepared at Tel Aviv University, under the supervision of Dr. A. J. Lazar.

Copyright @ 1974, American Mathematical Society 
$-y+a+x \in S_{2} \cap S_{3}$. Hence they have a nonempty intersection, and any $b \in S_{1} \cap S_{2} \cap S_{3}$ has all the desired properties.

Theorem. Let the scalars be real, and let $E$ be a Banach space with the 3.2.I.P. and let $K$ be as above. Then an element $\phi$ of $A_{0}(K, E)$ is extreme in the unit ball of this space if and only if $\phi(\operatorname{ext} K) \subset \operatorname{ext} S(E)$.

Proof. Since one direction is immediate, assume $\phi$ to be extreme. We make use of a selection theorem of Lazar and Lindenstrauss [3]. Define a set-valued mapping $\Sigma: K \rightarrow 2^{S(E)}$ by

$$
\Sigma(\mu)=\{x \in S(E) ;\|\phi(\mu) \pm x\| \leq 1\}, \quad \mu \in K .
$$

Now, for each $\mu \in K, \Sigma(\mu)$ is a norm-closed convex nonvoid subset of $S(E)$. Also $\Sigma(-\mu)=\Sigma(\mu)=-\Sigma(\mu)$ for each $\mu \in K$ (thus $\Sigma$ is symmetric), and for each $\mu_{1}, \mu_{2} \in K, 0 \leq \alpha . \leq 1$, we have

$$
\alpha \Sigma\left(\mu_{1}\right)+(1-\alpha) \Sigma\left(\mu_{2}\right) \subset \Sigma\left(\alpha \mu_{1}+(1-\alpha) \mu_{2}\right)
$$

(thus $\Sigma$ is convex). $\Sigma$ is also lower semicontinuous (in the sense of Michael [5]) with the $w^{*}$-topology on $K$ and the norm-topology on $S(E)$ : Let $\mu \in K$, $x \in \Sigma(\mu)$, and $\mu_{\alpha} \stackrel{w^{*}}{\rightarrow} \mu$ in $K$. We have to show the existence of $x_{\alpha} \in \Sigma\left(\mu_{\alpha}\right)$, for each $\alpha$, such that $x_{a} \rightarrow x$ in norm. But this is immediate since $\phi\left(\mu_{a}\right) \rightarrow$ $\phi(\mu)$ in norm, and, for each $\alpha$, the Lemma provides us with a $x_{\alpha} \in E$ such that $\left\|\phi\left(\mu_{\alpha}\right) \pm x_{\alpha}\right\| \leq 1$ (hence $x_{\alpha} \in \Sigma\left(\mu_{\alpha}\right)$ ) and $\left\|x_{a}-x\right\| \leq\left\|\phi\left(\mu_{\alpha}\right)-\phi(\mu)\right\|$. Hence the lower semicontinuity of $\Sigma$ is obvious. Now, assume that $\phi($ ext $K) \not \subset$ ext $S(E)$. Hence, there is a $\mu_{0} \epsilon$ ext $K$ and a nonzero element $x_{0}$ of $\Sigma\left(\mu_{0}\right)$. Now, $f\left(\alpha \mu_{0}\right)=\alpha x_{0}, \alpha \in[-1,1]$, is a $w^{*}$-continuous affine symmetric selection of $\Sigma$ restricted to $\operatorname{conv}\left(\left\{\mu_{0}\right\} U-\left\{\mu_{0}\right\}\right)$, for which $\left\{\mu_{0}\right\}$ is obviously essentially closed (cf. [3] for proper definitions). Hence, there is a $w^{*}$-continuous affine symmetric selection $\psi \in A_{0}(K, E)$ of $\Sigma$, such that $\psi\left(\mu_{0}\right)=x_{0} \neq 0$. Hence, for each $\mu \in K$ one has $\|\phi(\mu) \pm \psi(\mu)\| \leq 1$ and therefore $\|\phi \pm \psi\| \leq 1$. Since $\phi$ is extreme we must have $\psi=0$, a contradiction which completes the proof.

Remark. The estimate $\left\|x_{\alpha}-x\right\| \leq\left\|\phi\left(\mu_{\alpha}\right)-\phi(\mu)\right\|$, and therefore also the requirement that $E$ will have the 3.2.I.P., is apparently too strong for the proof of the Theorem and weaker assumptions will do as well.

\section{REFERENCES}

1. H. Fakhoury, Préduaux de L-espaces et éléments extrémaux, C. R. Acad. Sci. Paris Sér. A-B 272 (1971), A1703-A1706. MR 43 \#6700. 
2. A. J. Lazar, Affine functions on simplexes and extreme operators, Israel J. Math. 5 (1967), 31-43. MR 35 \#2128.

3. A. J. Lazar and J. Lindenstrauss, Banach spaces whose duals are $L_{1}$ spaces and their representing matrices, Acta Math. 126 (1971), 165-193. MR 45 \#862.

4. J. Lindenstrauss, Extension of compact operators, Mem. Amer. Math. Soc. No. 48 (1964)。. MR 31 \#3828.

5. E. Michael, Continuous selections. I, Ann. of Math. (2) 63 (1956), 361-382. MR 17, 990.

DE PARTMENT OF MATHEMATICAL SCIENCES, TEL AVIV UNIVERSITY, TEL AVIV, ISRAEL 